\title{
Convergence of two-dimensional hypergeometric series for algebraic functions
}

\author{
A. N. Cherepanskiy ${ }^{1}$ and A. K. Tsikh ${ }^{2}$ \\ ${ }^{1,2}$ Institute of Mathematics and Computer Science, Siberian Federal University, Pr. \\ Svobodnyi 79, Krasnoyarsk, Russia
}

\section{ARTICLE HISTORY}

Compiled April 13, 2020

\begin{abstract}
Description of convergence domains for multiple power series is a quite difficult problem. In 1889 J.Horn showed that the case of hypergeomteric series is more favorable. He found a parameterization formula for surfaces of conjugative radii of such series. But until recently almost nothing was known about the description of convergence domains in terms of functional inequalities $\rho_{j}\left(\left|a_{1}\right|, \ldots,\left|a_{m}\right|\right)<0$ relatively moduli $\left|a_{i}\right|$ of series variables. In this paper we give a such description for hypergeometric series representing solutions to tetranomial algebraic equations. In our study we use the remarkable observation by M. Kapranov (1991) consisting in the fact that the Horn's formulae give a parameterization of discriminant locus for a corresponding A-discriminant. We prove that usually the considered convergence domains are determined by a signle or two inequalities $\rho\left(\left|a_{t}\right|,\left|a_{s}\right|\right) \lessgtr 0$, where $\rho$ is a reduced discriminant.
\end{abstract}

\section{KEYWORDS}

Hypergeometric series, algebraic equations, Horn-Kapranov parameterization, amoeba

\section{AMS CLASSIFICATION \\ 33C $70,32 \mathrm{~A} 07$}

\section{Introduction}

Computation of convergence domains for a multiple power series is an actual and quite difficult problem. According to Abels lemma the convergence domains of these series are polycircular, i.e. they are determined by conditions on absolute values of variables. The domains of convergence for the series in one variable are disks. Moreover, the radii of these disks are defined in terms of series coefficients by Cauchy-Hadamard formula. For the series in several variables the corresponding Cauchy-Hadamard statement says only about what condition must the conjugate radii of convergence and coefficients of the series satisfy. However, this statement does not give an explicit formula (in terms of equations or parameterizations of surfaces boundary) for the conjugate radii of convergence and, hence, the boundary of convergence domain. 
The case of hypergeometric series is more favorable. Here the surface of the conjugate radii of convergence is obtained as envelope surface for the family of hyperplanes. In logarithmic scale these hyperplanes bound the convergence domains for diagonal subseries of given series (see Horn, [1]). Moreover, the parameterization of envelope surface is simple defined by the rational functions in definition of hypergeometric series. At the begining of the last century, it turned out that general algebraic functions belong to the class of hypergeometric-type functions (see $[2,3]$ ). Therefore, for these functions the problem of computation of convergence domains for the representing power series is not so hopeless. And this is confirmed by the result of this article.

In this paper we investigate the convergence domains of power series for a general algebraic function. By them one means (multivalued) solution $y=y(a)$ of the general equation

$$
a_{0}+a_{1} y+\ldots+a_{n-1} y^{n-1}+a_{n} y^{n}=0,
$$

with complex independent variable coefficients $a=\left(a_{0}, \ldots, a_{n}\right)$. In [2] Birkeland remarked that it is enough to study the reduced equation

$$
a_{0}+a_{1} y+\ldots+y^{p}+\ldots+y^{q}+\ldots+a_{n} y^{n}=0,
$$

obtained from the general equation (1.1) by fixing any pair of coefficients.

Following Sturmfels [4], with the equation (1.1) we associate the matrix:

$$
A=\left(\begin{array}{cccccc}
1 & 1 & 1 & \ldots & 1 & 1 \\
0 & 1 & 2 & \ldots & n-1 & n
\end{array}\right) .
$$

The kernel $A^{-1}(0)$ of the corresponding linear mapping is of dimension $n-1$. For any given choice of integers $0 \leq p<q \leq n$ a basis for this kernel can be composed by integer vectors

$$
(q-p) e_{\nu}+(\nu-q) e_{p}+(p-\nu) e_{q}, \quad \nu \neq p, q,
$$

with $e_{0}, \ldots, e_{n}$ being the standart basic vectors in $\mathbb{R}^{n+1}$.

Denote by $B_{p q}$ the matrix of size $(n+1) \times(n-1)$

$$
B_{p q}=\left(\beta_{\mu}^{\nu}\right),
$$

whose column $\beta^{\nu}$ are the basic vectors (1.3). This matrix defines so called Gale transform.

It is clear that $A B_{p q}=0$, and the square matrix obtained by deleting the rows $\beta_{p}$ and $\beta_{q}$ numbered $p$ and $q$ from $B_{p q}$ is equal to product $q-p$ and the identity matrix of size $n-1$.

In [2] was shown that in a neighbourhood of $a_{0}=0, \ldots[p] \ldots[q] \ldots, a_{n}=0$ the equation (1.2) defines $q-p$ analytic solutions $y(a)$ which are represented as the following hypergeometric-type series:

$$
\sum_{k \in \mathbb{N}^{n-1}} \frac{\varepsilon^{-\left\langle\beta_{q}, k\right\rangle+1}}{(q-p) k !} \frac{\Gamma\left(\left(-\left\langle\beta_{q}, k\right\rangle+1\right) /(q-p)\right)}{\Gamma\left(1+\left(\left\langle\beta_{p}, k\right\rangle+1\right) /(q-p)\right)} a_{0}^{k_{0}} a_{1}^{k_{1}} \ldots[p] \ldots[q] \ldots a_{n}^{k_{n}},
$$


where $\varepsilon$ runs over all $q-p$ values of the radical $(-1)^{\frac{1}{q-p}}$, and $\beta_{p}, \beta_{q}$ are vectors of the matrix $B_{p q}$.

We shall denote this series by $\sigma_{p q}$ even though it really does depend also on a choice of radical $\varepsilon$, which coincides with its value at the origin. The particular choice of $\varepsilon$ will not influence the convergence properties of $\sigma_{p q}$. Whenever $q-p=1$ the series is truly hypergeometric in the sense of Horn (see section 5). As was pointed out by Birkeland [2], one can regroup (1.4) as a sum of $(q-p)^{n-1}$ hypergeometric series by simply taking the equivalence classes of each index $k_{v}$ modulo $q-p$. We shall here for convenience be referring to $\sigma_{p q}$ itself as a hypergemetric series.

Domains of convergence of hypergeometric series were studied by Horn in his paper [1]. He described them for series of 2 and 3 variable. His description is based on computations of domains for diagonal series using D'Alembert's principle and taking intersections of these domains.

Next in [5] (see also [6]) there was obtained a combinatorial description of the convergence domains of the series (1.4) representing solutions of the reduced equations (1.2). This description is given in terms of reciprocal positions between convergence domain and connected components of the amoeba complement for the discriminant set (see section 4). The counterpart of this description was given in [7] in terms of analytic coninuation of a given power series by Poisceaux series converging in some other connected components of the given amoeba. The accurate definition of an amoeba for algebraic set is presented in the section 2 .

In [5] there were considered some tetranomial algebraic equations and was determined method for computation of the convergence domains. Unfortunately, in one of examples in [5] there was an incorrectness that we found out in conversation with Sateesh R. Mane. We would like to thank him for the relevant remarks.

The goal of the present study is to describe the convergence domains $D_{p q}$ of the hypergeometric-type series representing the solutions of a tetranomial equation. The main aim is to describe them in terms of system of functional inequalities.

By tetranomial equation we will mean the equation

$$
a_{0}+a_{l} y^{l}+a_{m} y^{m}+a_{n} y^{n}=0,
$$

with $l, m, n$ being relatively prime and $l<m<n$. It is known that the discriminant $\Delta$ of the complete equation (1.1) is defined by formula $a_{n}^{-1} R\left(f, f^{\prime}\right)$, where $R$ is the resultant of polynomial $f$ (from the left part of (1.1)) and its derivative $f^{\prime}$. Using representation of $R\left(f, f^{\prime}\right)$ by the Sylvester determinant, one can easily show that $R$ becames divisible by $a_{n}^{n-m} a_{0}^{l-1}$. Therefore it is sensibly to define the discriminant $\Delta(a)=\Delta\left(a_{0}, a_{l}, a_{m}, a_{n}\right)$ of equation (1.5) by formula

$$
\Delta(a)=\frac{1}{a_{n}^{n-m} a_{0}^{l-1}} R\left(f, f^{\prime}\right) .
$$

This discriminant possesses the following bi-homogeneity property

$$
\Delta\left(\lambda_{0} a_{0}, \lambda_{0} \lambda_{1}^{l} a_{l}, \lambda_{0} \lambda_{1}^{m} a_{m}, \lambda_{0} \lambda_{1}^{n} a_{n}\right)=\lambda_{0}^{n+m-l} \lambda_{1}^{m n} \Delta(a) .
$$

The reduction of the equation (1.5) can be obtained by fixing two coefficients $a_{p}$, $a_{q}$, with the pair $p, q \in\{0, l, m, n\}$. Denote the complementary pair by $t, s$. In the case when $a_{p}=a_{q}=1$, we denote the reduced discriminant $\left.\Delta\right|_{a_{p}=1, a_{q}=1}$ by $\Delta_{p q}\left(a_{t}, a_{s}\right)$. 
Solutions to this reduced equation are represented by hypergeometric-type double power series (1.4). It is known that the power series converge up to the first singularity of the functions, which they represent. Therefore, since the singular set of the solution $y(a)$ to reduced equation is completly defined by a zero set of the reduced discriminant, it is reasonable to describe the convergence domain of series by functional inequalities using this discriminant.

We study the convergence domains $D_{p q}$ of series (1.4) representing the solution $y\left(a_{s}, a_{t}\right)$ of the distinguished reduced equation (1.5) (when $a_{p}=q_{q}=1$ ).

Theorem 1.1. Except for the domains $D_{0 l}$ and $D_{m n}$ with odd $l$, odd $m$, and even $n$, the domains $D_{p q}$ are determined by one or two inequalities of type

$$
\left.\Delta\right|_{a_{p}= \pm 1, a_{q}= \pm 1}\left(a_{s}, a_{t}\right) \lessgtr 0 .
$$

Specified inequalities are given in Statements $6.1-6.6$, where instead $\left(a_{s}, a_{t}\right)$ we write $(a, b)$. For example, consider the reduced tetranomial equation

$$
1+a y+y^{2}+b y^{7}=0
$$

with $[p, q]=[0,2]$. According to $(1.4)$ near the origin $(a, b)=(0,0)$ this equation has two solutions represented by the following series

$$
y(a, b)=\frac{1}{2} \sum_{k \in \mathbb{N}^{2}} \frac{\varepsilon^{-\left\langle\beta_{2}, k\right\rangle+1}}{k !} \frac{\Gamma\left(\left(-\left\langle\beta_{2}, k\right\rangle+1\right) / 2\right)}{\Gamma\left(1+\left(\left\langle\beta_{0}, k\right\rangle+1\right) / 2\right)} a^{k_{1}} b^{k_{2}}
$$

with $\beta_{0}=\left(-\frac{1}{2}, \frac{5}{2}\right), \beta_{2}=\left(-\frac{1}{2},-\frac{7}{2}\right)$, and $\varepsilon= \pm i$. The convergence domain $D_{02}$ is of type $D_{0 m}$, therefore we are in position of the Statement 6.3 with $l=1, m=2$ and $n=7$. Due to the Statement 6.3 we have

$$
D_{02}=\{\Delta(1,|a|,-1,|b|)<0\} \cap\{\Delta(1,-|a|, 1,-|b|)>0\} .
$$

The computation of the discriminant $\Delta$ according to representation (1.6) allows us to define $D_{02}$ by the pair of explicit inequalities:

$$
\begin{aligned}
& 46656|a|^{7}|b|+381024|a|^{5}|b|+926100|a|^{3}|b|-3125|a|^{2}+600250|a||b|+823543|b|^{2}-12500<0, \\
& -46656|a|^{7}|b|+381024|a|^{5}|b|-926100|a|^{3}|b|+3125|a|^{2}+600250|a||b|-823543|b|^{2}-12500<0 .
\end{aligned}
$$

In the logarithmic scale the shape of $D_{02}$ is depicted by figure 4 as a shaded domain. We see that the boundary of the shaded domain consists of two curves which correspond to different inequalities.

Remark that the statement of the Theorem 1.1 one can use also to describe the convergence domains for transcendental hypergeometric series satisfying the Mellin system of differential hypergeometric equations introduced in [3]. Hypergeometric series for this system were studied in the paper [8]. Note that firstly the series for the solutions of algebraic equation were obtained by Mellin in terms of Mellin-Barns integrals (about these integrals with applications to the algebraic function theory, see [9]-[11]).

Along with the discriminant in our investigation will be used the following ingredients: Newton polytope, amoeba and its contour, Horn-Kapranov parameterization for 
discriminant locus. Finally note that all these tools are fruitful for study the multiple roots of algebraic equations (see [12]).

\section{The Newton polytope for the discriminant}

Definition 2.1. For a given polynomial

$$
f(z)=\sum_{k \in A \subset \mathbb{Z}^{d}} c_{k} z_{1}^{k_{1}} \ldots z_{d}^{k_{d}} \in \mathbb{C}\left[z_{1}, \ldots, z_{d}\right]
$$

its Newton polytope $\mathcal{N}_{f}$ is defined as the convex hull in $\mathbb{R}^{d}$ of the set $A \subset \mathbb{Z}^{d} \subset \mathbb{R}^{d}$.

Denote $\mathbb{C} \backslash\{0\}$ by $\mathbb{C}^{*}$ and consider the mapping Log $:\left(\mathbb{C}^{*}\right)^{d} \rightarrow \mathbb{R}^{d}$ :

$$
\log :\left(z_{1}, \ldots, z_{d}\right) \rightarrow\left(\log \left|z_{1}\right|, \ldots, \log \left|z_{d}\right|\right) .
$$

Definition 2.2. [13, p.194] The amoeba of an algebraic set $V \subset\left(\mathbb{C}^{*}\right)^{d}$ is the image $\log V$, which is denoted by $\mathcal{A}_{V}$.

If $V$ is a hypersurface defined by polynomial $f(z)$ we also write $\mathcal{A}_{V}=\mathcal{A}_{f}$. It is known [13] that the complement $\mathbb{R}^{d} \backslash \mathcal{A}_{f}$ consists of a finite connected components which are open and convex.

In our investigation we are interested the case when $f$ is the discriminant $\Delta=\Delta(a)$ of the polynomial in equation (1.1). It is known that the Newton polytope $\mathcal{N}$ of $\Delta$ is combinatorially equivalent to the $(n-1)$-dimensional cube ([see 13, p.412]).

It will be of importance for our investigations that there exist natural bijections between the following sets (see Ch. 12, Theorems 2.2 and 2.3 in [13], and [14]):

$$
\text { \{subdivisions of }[0, n]\} \leftrightarrow\left\{\text { vertices of } \mathcal{N}_{\Delta}\right\} \leftrightarrow\left\{\text { components of } \mathbb{R}^{n+1} \backslash \mathcal{A}_{\Delta}\right\} \text {. }
$$

Let us explain the first bijection between subdivisions and vertices in some detail. It is a special instance of a result from [13] relating triangulations of certain polytopes (in our case the segment $[0, n]$ ) to extremal monomials in general discriminants (in our case in the discriminant $\Delta$ ). To any given subdivision $\left[0, i_{1}\right],\left[i_{1}, i_{2}\right], \ldots,\left[i_{s}, n\right]$, obtained by an integer subset $I=\left\{i_{1}<i_{2}<\ldots<i_{s}\right\}$, one assigns the vector $k_{I}=\left(k_{0}, k_{1}, \ldots, k_{n}\right)$ with coordinates

$$
\begin{gathered}
k_{0}=i_{1}-i_{0}-1, \quad k_{n}=i_{s+1}-i_{s}-1, \\
k_{i_{q}}=i_{q+1}-i_{q-1}, i_{q} \in I, \\
k_{i}=0, i \notin I .
\end{gathered}
$$

Let $l_{q}=i_{q+1}-i_{q}(0 \leqslant q \leqslant s)$. Thus, the vertex monomial

$$
a^{k_{I}}=a_{0}^{l_{0}-1} a_{i_{1}}^{l_{1}+l_{0}} a_{i_{2}}^{l_{2}+l_{1}} \ldots a_{i_{s}}^{l_{s}+l_{s-1}} a_{n}^{l_{s}-1}
$$


appears in $\Delta$ with the coefficient

$$
c_{k_{I}}:=c_{I}:=(-1)^{n(n-1) / 2} \prod_{q=0}^{s}(-1)^{\frac{l_{q}\left(l_{q}-1\right)}{2}} l_{q}^{l_{q}} .
$$

In the case $n=3$ there are four subdivisions

$$
\{[0,3]\} ;\{[0,1],[1,3]\} ;\{[0,2],[2,3]\} ;\{[0,1],[1,2],[2,3]\}
$$

with associated vertices

$$
(2,0,0,2),(0,3,0,1),(1,0,3,0),(0,2,2,0)
$$

and corresponding monomials $27 a_{0}^{2} a_{3}^{2}, 4 a_{1}^{3} a_{3}, 4 a_{0} a_{2}^{3},-a_{1}^{2} a_{2}^{2}$. Remark that the full discriminant of the cubic equation $a_{0}+a_{1} y+a_{2} y^{2}+a_{3} y^{3}=0$ is equal

$$
\Delta=27 a_{0}^{2} a_{3}^{2}+4 a_{1}^{3} a_{3}+4 a_{0} a_{2}^{3}-18 a_{0} a_{1} a_{2} a_{3}-a_{1}^{2} a_{2}^{2} .
$$

\section{Reduced discriminant, its parameterization and amoeba}

The discriminant of the reduced equation (1.2) we denote $\Delta_{p q}$ and call it a reduced discriminant. Clearly $\Delta_{p q}$ is obtained from $\Delta(a)$ by substitution $a_{p}=a_{q}=1$.

The zero set of $\Delta_{p q}$ (we call it a discriminant set) is parameterized by a mapping

$$
\psi_{p q}(s): \mathbb{C P}^{n-2} \rightarrow \mathbb{C}^{n-1}
$$

from projective space with homogeneous coordinates $s=\left(s_{1}: \ldots: s_{n-1}\right)$ into the space of coefficients $\left(a_{0}, \ldots[p] \ldots[q] \ldots, a_{n}\right)$ of the equation (1.2). This parameterization is defined by formula

$$
\psi_{p q}(s)=\left(\prod_{j=0}^{n}\left\langle\beta_{j}, s\right\rangle^{\beta_{j}^{0} /(q-p)}, \ldots[p] \ldots[q] \ldots, \prod_{j=0}^{n}\left\langle\beta_{j}, s\right\rangle^{\beta_{j}^{n} /(q-p)}\right) .
$$

with the vectors $\beta_{0}, \ldots, \beta_{n}$ being the row vectors of the matrix $B_{p q}$. More precisely, in view of (1.3), we may rewrite the $\nu$ th component of $\psi_{p q}$ as

$$
a_{\nu}=\frac{(q-p) s_{\nu}}{\left\langle\beta_{p}, s\right\rangle}\left(\frac{\left\langle\beta_{p}, s\right\rangle}{\left\langle\beta_{q}, s\right\rangle}\right)^{\frac{\nu-p}{q-p}}
$$

Thus the multi-valuedness depends only on the choice of the radical $\left(\left\langle\beta_{p}, s\right\rangle /\left\langle\beta_{q}, s\right\rangle\right)^{1 /(q-p)}$. Therefore, $\psi_{p q}$ is a one-to- $(q-p)$ mapping.

The Newton polytope for the reduced discriminant $\Delta_{p q}$ is also combinatorially equivalent to the $(n-1)$-dimensional cube. An important role are played by the amoeba of the reduced equation and, in particular, the notion of the amoeba's contour. We denote the zero set of the reduced discriminant by $\nabla_{p q}$.

Definition 3.1. [15] The set of critical values of the mapping $\log : \nabla_{p q} \rightarrow \mathcal{A}_{\nabla_{p q}}$ is called the contour of the amoeba $\mathcal{A}_{\nabla_{p q}}$. 
The contour contains the boundary of the amoeba and may cross its interior (for instance, see fig. 2, where the cuspidal part of the contour crosses the interior of the amoeba).

There is an explicit parameterization of the amoeba contour.

Proposition 3.2. [5] The contour of the amoeba $\mathcal{A}_{\nabla_{p q}}$ coincides with the image of the projective space $\mathbb{R P}^{n-2}$ under the composed mapping Log $\circ \psi_{p q}$.

\section{Combinatorial description of convergence domains $D_{p q}$}

The following theorem gives a combinatorial description of the convergence domain in terms of the connected component of the amoeba complement $\mathbb{R}^{n-1} \backslash \mathcal{A}_{\Delta_{p q}}$.

Theorem 4.1. [5] The domain of convergence $D_{p q}$ of the series (1.4) is a complete Reinhardt domain such that the corresponding convex domain $\log \left(D_{p q}\right)$ contains all the connected components of the amoeba complement $\mathbb{R}^{n-1} \backslash \mathcal{A}_{\Delta_{p q}}$ that are associated with subdivisions of $[0, n]$ containing the segment $[p, q]$, while it is disjoint from all the other complement components.

A similar statement holds not only for the complete equation (1.2) (where all exponents from $[0, n]$ present) but also for the sparse equation

$$
a_{0}+a_{1} y^{d_{1}}+\ldots+y^{d_{p}}+\ldots+y^{d_{q}}+\ldots+a_{k} y^{d_{k}}=0
$$

with $0=d_{0}<d_{1} \ldots<d_{k-1}<d_{k}=n$.

In this case we need that $\operatorname{gcd}\left(d_{1}, \ldots, d_{k}\right)=1$. As before the reduction of the sparse equation is obtained by fixing any pair of coefficients. Furthermore the subdivisions of the sparse seqment are realized by means of verticies $0, d_{1}, \ldots, d_{k-1}, n$. In the case of tetranomial sparse equation there are six subdivisons and hence six domains $D_{p q}$. It will be convenient for us to divide these domains into three pairs $\left(D_{0 n}, D_{l m}\right),\left(D_{0 m}, D_{l n}\right)$ and $\left(D_{0 l}, D_{m n}\right)$.

As a comment to Theorem 4.1 let us consider the domain $D_{01}$ for the cubic equation. In the logarthmic scale this domain is depicted as the shaded domain on figure 6 . This domain contains two connected components of the complement of amoeba $\mathcal{A}_{\Delta_{01}}$ associated with subdivisions of $[0,3]$

$$
\{[0,1],[1,2],[2,3]\} \text { and }\{[0,1],[1,3]\}
$$

containing the segment $[0,1]$ (see figure 1 ).

\section{Horn's theorem in two-dimensional case}

In his paper [1], Horn presented a description of convergence domains for a hypergeometric series in 2 and 3 variables. He considered the positive octants $\mathbb{Z}_{+}^{2}$ and $\mathbb{Z}_{+}^{3}$ as an array of summing. Let us describe the Horn's result for the series in 2 variables

$$
H\left(x_{1}, x_{2}\right)=\sum_{k_{1}, k_{2} \geqslant 0} \varphi\left(k_{1}, k_{2}\right) x_{1}^{k_{1}} x_{2}^{k_{2}} .
$$




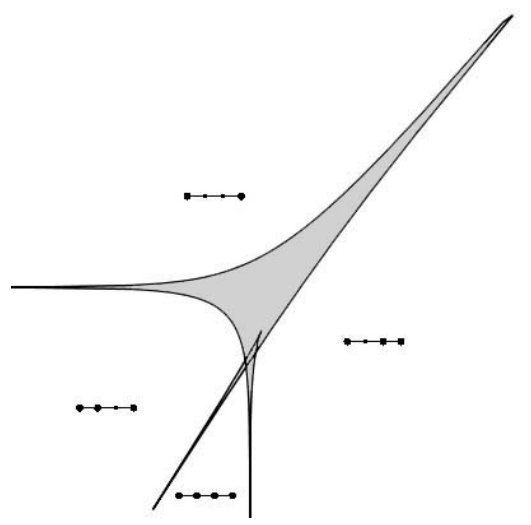

Figure 1. Amoeba of the cubic discriminant $\Delta_{01}$ and connected components of the amoeba complement.

By the Horn's definition this series is hypergeometric if the relations

$$
R_{1}\left(k_{1}, k_{2}\right):=\frac{\varphi\left(k_{1}+1, k_{2}\right)}{\varphi\left(k_{1}, k_{2}\right)}, \quad R_{2}\left(k_{1}, k_{2}\right):=\frac{\varphi\left(k_{1}, k_{2}+1\right)}{\varphi\left(k_{1}, k_{2}\right)}
$$

are rational functions of variables $k_{1}$ and $k_{2}$. In [1] were introduced the limits

$$
\Phi_{1}\left(s_{1}, s_{2}\right)=\lim _{l \rightarrow \infty} R_{1}\left(s_{1} l, s_{2} l\right), \quad \Phi_{2}\left(s_{1}, s_{2}\right)=\lim _{l \rightarrow \infty} R_{2}\left(s_{1} l, s_{2} l\right)
$$

and noted that the functions $\Phi_{i}$ are rational and have a zero homogeneity property. In other words, this functions depend on the relation $s=s_{1}: s_{2}$ only. Using them, one can compute the domain of convergence $G$ for the series $H\left(x_{1}, x_{2}\right)$.

Theorem 5.1. [1] If the point $\left(x_{1}^{0}, x_{2}^{0}\right)$ lies outside of the bicylinder

$$
\Delta=\left\{\left|x_{1}\right|<\left|\frac{1}{\Phi_{1}(1,0)}\right|,\left|x_{2}\right|<\left|\frac{1}{\Phi_{2}(0,1)}\right|\right\},
$$

or for some positive direction $s_{1}: s_{2}$

$$
\left|x_{1}^{0}\right|>\left|\frac{1}{\Phi_{1}\left(s_{1}, s_{2}\right)}\right|, \quad\left|x_{2}^{0}\right|>\left|\frac{1}{\Phi_{2}\left(s_{1}, s_{2}\right)}\right|
$$

then the power series $H\left(x_{1}, x_{2}\right)$ diverges at the point $\left(x_{1}^{0}, x_{2}^{0}\right)$.

If the point $\left(x_{1}^{0}, x_{2}^{0}\right)$ lies inside of the bicylinder $\Delta$ and for every positive direction $s_{1}: s_{2}$ at least one of the inequalities is satisfied

$$
\left|x_{1}^{0}\right|<\left|\frac{1}{\Phi_{1}\left(s_{1}, s_{2}\right)}\right|, \quad\left|x_{2}^{0}\right|<\left|\frac{1}{\Phi_{2}\left(s_{1}, s_{2}\right)}\right|,
$$

then the power series $H\left(x_{1}, x_{2}\right)$ converges at the point $\left(x_{1}^{0}, x_{2}^{0}\right)$. 
The following statement follows from the important and deep observation by M. Kapranov about the relation between the singularities of hypergeometric functions and discriminants (see [16]).

Theorem 5.2. [5] For the hypergeometric type series (1.4) the functions $\frac{1}{\Phi_{i}}$ coincide with the components of the mapping $\psi_{p q}$ defined by (3.1).

Thus Horn's parameterisation of the convergence domain boundary for the hypergeometric series coincides with the parameterization of the discriminant set. Therefore we call the mapping $\psi_{p q}: \mathbb{C} \mathbb{P}^{n-2} \rightarrow \mathbb{C}^{n-1}$ the Horn-Kapranov parameterization.

In accordance with Theorem 5.1 and Theorem 5.2 the Log-images of the convergance domains $D_{p q}$ andjoin amoebas $\mathcal{A}_{\Delta_{p q}}$ and their contours as shaded domains in figures $2-7$.

\section{Proof of the Theorem 1.1}

For the reduction of equation (1.5) by fixing a pair $[p, q]$ we will obtain the system of inequalities that describe the corresponding convergence domain $D_{p q}$ of the solution $y=y\left(a_{t}, a_{s}\right)$. For this purpose we use the parameterisation $\psi_{p q}$ of the discriminant locus $\left\{\Delta_{p q}\left(a_{t}, a_{s}\right)=0\right\}$ given by (3.1). Remark that this locus is a singular set of the algebraic function $y\left(a_{t}, a_{s}\right)$.

By Proposition 3.2, the contour $\mathcal{C}_{p q}$ of the amoeba $\mathcal{A}_{p q}$ coincides with the image $\log \circ \psi_{p q}\left(\mathbb{R P}^{1}\right)$. The Horn's Theorem 5.1 and Theorem 5.2 imply that the restriction $\psi_{p q}$ on $\mathbb{R}_{+} \subset \mathbb{R P}^{1}$ yields the part of the contour $\mathcal{C}_{p q}$ describing the domain $D_{p q}$. Since the convergence domain of a power series is a polycircular domain, we need to use in the description of $D_{p q}$ only the absolute values $\left|a_{t}\right|,\left|a_{s}\right|$. The main point consists of reflection of the nearest singular points of the solution $y\left(a_{t}, a_{s}\right)$ into the positive quadtrant of the real part $\mathbb{R}^{2}$ in the complex space $\mathbb{C}^{2}$ of variables $a_{t}, a_{s}$.

Let us show the idea by a simple example of the cubic reduced trinomial equation:

$$
y^{3}+a y+1=0 .
$$

The discriminant $\Delta(a)=4 a^{3}+27$ of this equation has no positive roots, therefore $\Delta(|a|)$ is everywhere positive. It follows that we are not able to describe in terms of $\Delta(|a|)$ the convergence domain of the solution series

$$
y(a)=\frac{1}{3} \sum_{k=0}^{\infty} \frac{\Gamma\left(\frac{1}{3}+\frac{1}{3} k\right)}{k ! \Gamma\left(\frac{4}{3}+\frac{2}{3} k\right)} a^{k} .
$$

However, considering the function $\Delta(-|a|)=-4|a|^{3}+27$ we can describe the true domain of convergence $|a|<3 / \sqrt[3]{4}$ by the inequality $\Delta(-|a|)>0$. Therefore using the reflection procedure $\Delta(|a|) \rightarrow \Delta(-|a|)$ we arrive the solution of our question.

For the reduced tetranomial equation a corresponding reflection is applied to some branches of $\psi_{p q}$ restricted to $\mathbb{R}_{+}$from the list of branches of the map $\psi_{p q}$.

To define the reciprocal distribution between the image $\log D_{p q}$ and the contour of amoeba $\mathcal{A}_{\Delta_{p q}}$ we use the second bijection in (2.1). For that we compute the vertex monomials of the discriminant $\Delta$ for equation (1.5) and their coefficients, using formulae (2.2) and (2.3). 
For the general tetranomial equation (1.5) one has four subdivisons:

$$
I_{1}=\{[0, l],[l, n]\}, I_{2}=\{[0, m],[m, n]\}, I_{3}=\{[0, n]\}, I_{4}=\{[0, l],[l, m],[m, n]\} .
$$

Table 1. The vertices $k_{I}$ of $\mathcal{N}_{\Delta}$ and the corresponding coefficients $C_{k_{I}}$.

\begin{tabular}{|l|l|l|}
\hline Subdivisions & Vertices of $\mathcal{N}_{\Delta}$ & Coefficients \\
\hline$\{[0, l],[l, n]\}$ & $(l-1, n, 0, n-l-1)$ & $(-1)^{l(n-l)} l^{l}(n-l)^{n-l}$ \\
\hline$\{[0, m],[m, n]\}$ & $(m-1,0, n, n-m-1)$ & $(-1)^{m(m-n)} m^{m}(n-m)^{n-m}$ \\
\hline$\{[0, n]\}$ & $(n-1,0,0, n-1)$ & $n^{n}$ \\
\hline$\{[0, l],[l, m],[m, n]\}$ & $(l-1, m, n-l, n-m-1)$ & $(-1)^{l(m-l)+m(n-m)} l^{l}(n-m)^{n-m}(m-l)^{m-l}$ \\
\hline
\end{tabular}

\subsection{The convergence domain $D_{0 n}$}

The reduced equation for the pair $[p, q]=[0, n]$ has the following form:

$$
1+a y^{l}+b y^{m}+y^{n}=0 .
$$

Due to (10) in this case we have the following parameterization $\psi_{0 n}: \mathbb{C P}^{1} \rightarrow \mathbb{C}^{2}$ of the discriminant set $\nabla_{0 n}$ :

$$
\left\{\begin{array}{l}
a=\frac{n s}{(-n+l) s-n+m}\left(\frac{(-n+l) s-n+m}{-l s-m}\right)^{\frac{l}{n}} \\
b=\frac{n}{(-n+l) s-n+m}\left(\frac{(-n+l) s-n+m}{-l s-m}\right)^{\frac{m}{n}}
\end{array} .\right.
$$

Since $0<l<m<n$ the linear functions

$$
\alpha(s):=(-n+l) s-n+m, \quad \beta(s):=-l s-m
$$

are negative for all $s>0$. It follows that for $s \in \mathbb{R}_{+}$the multivalued map $\psi_{0 n}(s)$ has a real branch with values in the negative quadrant $\{a<0, b<0\} \subset \mathbb{R}^{2}$ (taking the positive value for the radical $(\alpha(s) / \beta(s))^{\frac{1}{n}}$ in $(6.1)$, we arrive this case). Using the reflection

$$
(a, b) \rightarrow(-a,-b)
$$

we move the image $\psi_{0 n}\left(\mathbb{R}_{+}\right)$under the mentioned branch into the positive quadrant $\{a>0, b>0\}$ of $\mathbb{R}^{2}$. The points of this image satisfy the equation

$$
\Delta_{0 n}(-|a|,-|b|)=0 .
$$

By statements of Theorems 5.1 and 5.2 we get that the boundary of the convergence domain $D_{0 n}$ satisfies this equation.

Since $D_{0 n}$ contains the origin $(a, b)=(0,0)$, the sign of $\Delta_{0 n}(-|a|,-|b|)$ in $D_{0 n}$ is defined by its constant term. The 3-rd row of the table 1 tells us that this term $n^{n}$ is positive. Thus we get the following statement. 
Statement 6.1. The convergence domain $D_{0 n}$ is a connected component of the set

$$
\left\{-\Delta_{0 n}(-|a|,-|b|)<0\right\},
$$

containing the origin $(a, b)=(0,0)$.

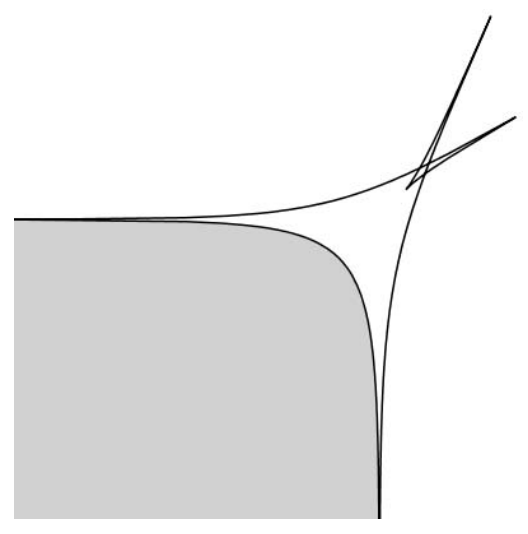

Figure 2. The Log-image shape of $D_{0 n}$ (shaded), and the amoebas contour for the discriminant $\Delta_{0 n}$

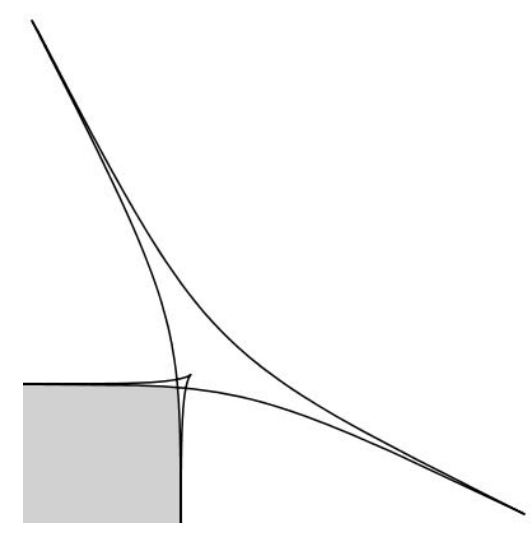

Figure 3. The Log-image shape of $D_{l m}$ (shaded), and the amoebas contour for the discriminant $\Delta_{l m}$

\subsection{The convergence domain $D_{l m}$}

The reduced equation for the pair $[p, q]=[l, m]$ has the following form:

$$
a+y^{l}+y^{m}+b y^{n}=0 .
$$

We have the following parameterization $\psi_{l m}: \mathbb{C P}^{1} \rightarrow \mathbb{C}^{2}$ of its discriminant set $\nabla_{l m}$ :

$$
\left\{\begin{array}{l}
a=\frac{(m-l) s}{-m s+n-m}\left(\frac{-m s+n-m}{l s+l-n}\right)^{\frac{-l}{m-l}} \\
b=\frac{(m-l)}{-m s+n-m}\left(\frac{-m s+n-m}{l s+l-n}\right)^{\frac{n-l}{m-l}}
\end{array},\right.
$$

with linear functions

$$
\alpha(s):=-m s+n-m, \quad \beta(s):=l s+l-n
$$

under the radical sign. Unlike the previous case 6.1 , now we have two positive values of $s$ in which $\alpha(s)$ and $\beta(s)$ change the signs, namely, $s=\frac{n-m}{m}$ and $s=\frac{n-l}{l}$. It means that there are three intervals

$$
\left(0, \frac{n-m}{m}\right),\left(\frac{n-m}{m}, \frac{n-l}{l}\right),\left(\frac{n-l}{l}, \infty\right)
$$

in $\mathbb{R}_{+}$which we have to consider. Using the Theorem 5.1 and the shape of amoeba $\mathcal{A}_{\Delta_{l m}}$ (see fig. 3), we conclude that the boundary of $D_{l m}$ is described by the pieces 
of images $\psi_{l m}(s)$ where $s$ runs over two intervals $\left(0, \frac{n-m}{m}\right)$ and $\left(\frac{n-l}{l}, \infty\right)$. Indeed, the upper part of the convergence domain boundary corresponds to image $\psi_{l m}(s)$ with $s \in\left(0, \frac{n-m}{m}\right)$ and the right one corresponds to $\psi_{l m}(s)$ with $s \in\left(\frac{n-l}{l}, \infty\right)$.

In the case when $s$ runs over $\left(0, \frac{n-m}{m}\right)$, the map $\psi_{l m}(s)$ may not have real branches since the radicand in (6.2) is negative. At first we need to move its image to the real subspace by the reflection $(a, b) \rightarrow\left(\varepsilon^{-l} a, \varepsilon^{n-l} b\right)$ with $\varepsilon=(-1)^{\frac{1}{m-l}}$. Under this reflection we get a real branch with values in the negative quadrant $\{a<0, b<0\} \subset$ $\mathbb{R}^{2}$. Therefore by the reflection

$$
(a, b) \rightarrow\left(-\varepsilon^{-l} a,-\varepsilon^{n-l} b\right)
$$

we remove the image of the mentioned branch to the positive quadrant $(a>0, b>0)$ of $\mathbb{R}^{2}$. This image satisfies the equation

$$
\Delta_{l m}\left(-\varepsilon^{-l}|a|,-\varepsilon^{n-l}|b|\right)=0 .
$$

The same arguments show that in the case $s \in\left(\frac{n-l}{l}, \infty\right)$ we need to do the reflection

$$
(a, b) \rightarrow\left(\varepsilon^{-l} a, \varepsilon^{l-n} b\right)
$$

in order to get the next equation

$$
\Delta_{l m}\left(\varepsilon^{-l}|a|, \varepsilon^{n-l}|b|\right)=0
$$

for other part of the convergence domain boundary.

One can rewrite the discriminant in (6.3) and (6.4) using the bihomogeneity formalua (1.7). For instance, rewriting the discriminant in (6.4) as $\Delta\left(\varepsilon^{-l}|a|, 1,1, \varepsilon^{n-l}|b|\right)$, representing here the units: $1=\varepsilon^{-l} \varepsilon^{l} \cdot 1,1=\varepsilon^{-l} \varepsilon^{m} \cdot(-1)$, and taking in (1.7) $\lambda_{0}=\varepsilon^{-l}, \lambda_{1}=\varepsilon=(-1)^{\frac{1}{m-l}}, a_{0}=|a|, a_{l}=1, a_{m}=-1, a_{n}=|b|$, we get the identity

$$
\Delta_{l m}\left(\varepsilon^{-l}|a|, \varepsilon^{n-l}|b|\right)=\varepsilon^{-l(n+m-l)+m n} \Delta(|a|, 1,-1,|b|) .
$$

Therefore we get the following statement.

Statement 6.2. The convergence domain $D_{l m}$ is of type

$$
\{\Delta(|a|, 1,-1,|b|) \lessgtr 0\} \cap\{\Delta(-|a|, 1,-1,-|b|) \lessgtr 0\} .
$$

\subsection{The convergence domains $D_{0 m}$ and $D_{l n}$}

Here we do the computation only for the domain $D_{0 m}$ since the arguments for the case $D_{l n}$ are similar. The reduced equation for the pair $[0, m]$ has the following form:

$$
1+a y^{l}+y^{m}+b y^{n}=0
$$




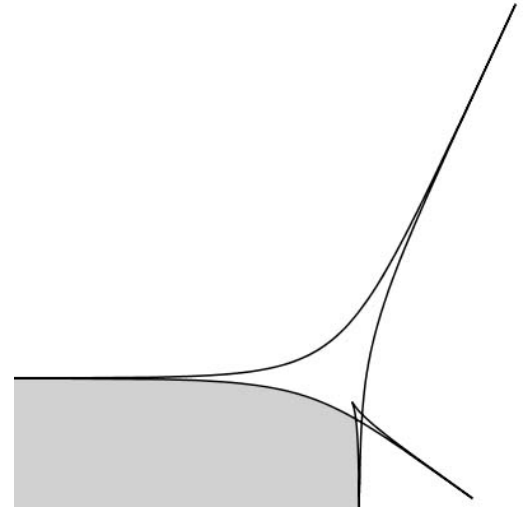

Figure 4. The Log-image shape of $D_{0 m}$ (shaded), and the amoebas contour for the discriminant $\Delta_{0 m}$

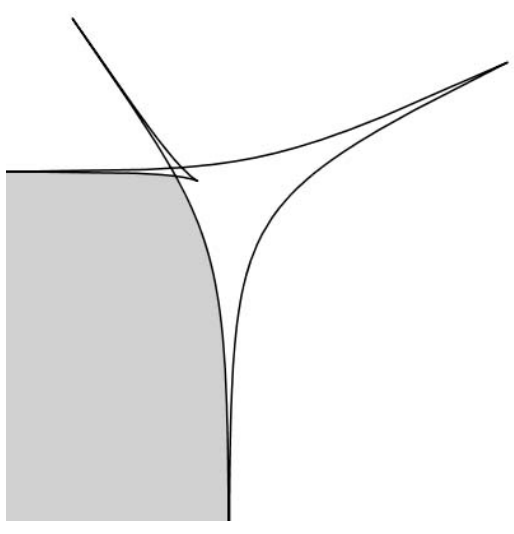

Figure 5. The Log-image shape of $D_{l n}$ (shaded), and the amoebas contour for the discriminant $\Delta_{l n}$

The parameterization of the discriminant set is the following:

$$
\psi_{0 m}:\left\{\begin{array}{l}
a=\frac{m s}{(-m+l) s+n-m}\left(\frac{(-m+l) s+n-m}{-l s-n}\right)^{\frac{l}{m}} \\
b=\frac{m}{(-m+l) s+n-m}\left(\frac{(-m+l) s+n-m}{-l s-n}\right)^{\frac{n}{m}} .
\end{array}\right.
$$

We have one positive value $s=\frac{n-m}{m-l}$ in which the linear functions

$$
\alpha(s):=(-m+l) s+n-m, \quad \beta(s):=-l s-n
$$

change the sign on interval $(0, \infty)$ and it means that there are two subintervals

$$
\left(0, \frac{n-m}{m-l}\right),\left(\frac{n-m}{m-l}, \infty\right)
$$

which we have to consider. Similarly to the subsection 6.2 we find the following reflections for these intervals: $\Delta_{0 m}\left(\varepsilon^{l}|a|, \varepsilon^{n}|b|\right)$ and $\Delta_{0 m}(-|a|,-|b|)$, where $\varepsilon$ is a primitive $\operatorname{root}(-1)^{1 / m}$. Using the Theorem 5.1 and the shape of amoeba $\mathcal{A}_{\Delta_{0} m}$ (see fig. 4), we get the corresponding equations $\left\{\Delta_{0 m}\left(\varepsilon^{l}|a|, \varepsilon^{n}|b|\right)=0\right\}$ and $\left\{\Delta_{0 m}(-|a|,-|b|)=0\right\}$ for the parts of the boundary $\partial D_{0 m}$.

Rewriting here the first discriminant as $\Delta\left(1, \varepsilon^{l}|a|, 1, \varepsilon^{n}|b|\right)$, and taking in (1.7) $\lambda_{0}=a_{0}=1, \lambda_{1}=\varepsilon=(-1)^{\frac{1}{m}}, a_{l}=|a|, a_{m}=-1, a_{n}=|b|$ we get the identity

$$
\Delta_{0 m}\left(\varepsilon^{l}|a|, \varepsilon^{n}|b|\right)=\varepsilon^{m n} \Delta(1,|a|,-1,|b|) .
$$

Thus we get the following statement.

Statement 6.3. The convergence domain $D_{0 m}$ is of type

$$
\{\Delta(1,|a|,-1,|b|) \lessgtr 0\} \cap\{\Delta(1,-|a|, 1,-|b|) \lessgtr 0\} .
$$

Symmetrically, one has the following result for the domain $D_{l n}$. 


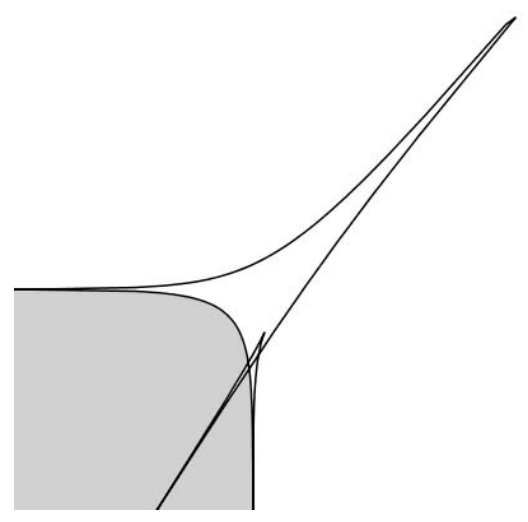

Figure 6. The Log-image shape of $D_{0 l}$ (shaded), and the amoebas contour for the discriminant $\Delta_{0 l}$

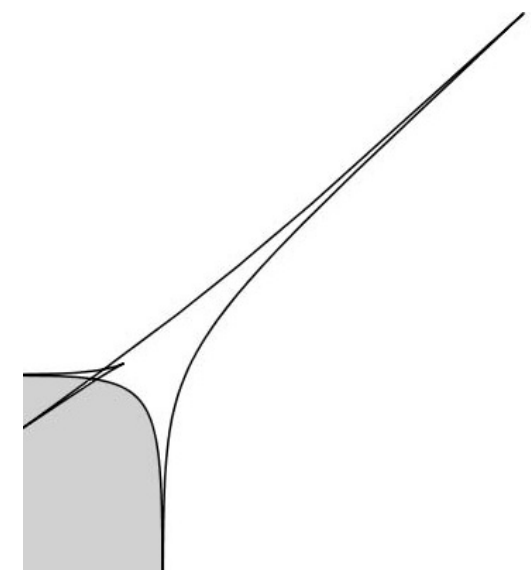

Figure 7. The Log-image shape of $D_{m n}$ (shaded), and the amoebas contour for the discriminant $\Delta_{m n}$

Statement 6.4. The convergence domain $D_{l n}$ is of type

$$
\{\Delta(|a|,-1,|b|, 1) \lessgtr 0\} \cap\{\Delta(-|a|, 1,-|b|, 1) \lessgtr 0\} .
$$

\subsection{The convergence domains $D_{0 l}$ and $D_{m n}$}

Similarly to the subsection 6.3 , we do the computaion only for the domain $D_{0 l}$. The reduced equation for the pair $[0, l]$ has the following form:

$$
1+y^{l}+a y^{m}+b y^{n}=0 .
$$

The parameterization of the discriminant set is the following:

$$
\psi_{0 l}:\left\{\begin{array}{rl}
a & =\frac{l s}{(m-l) s+n-l}\left(\frac{(m-l) s+n-l}{-m s-n}\right)^{\frac{m}{l}} \\
b & =\frac{l}{(m-l) s+n-l}\left(\frac{(m-l) s+n-l}{-m s-n}\right)^{\frac{n}{l}}
\end{array} .\right.
$$

Linear functions $\alpha(s)$ and $\beta(s)$ in (6.5) do not have zeros on $\mathbb{R}_{+}$. At the same time the singular points of the discriminant set are the images $\psi_{0 l}\left(s_{0}\right)$ of the point $s_{0} \in \mathbb{R}_{-}$ with respect to $l$-valued map $\psi_{0 l}$ (the Log-image of $\psi_{0 l}\left(s_{0}\right)$ corresponds to the cuspidal point of the amoeba contour in fig. 6). Therefore, by the Horn theorem, the boundary of the domain $D_{0 l}$ is determined by the parameterization $(|a(s)|,|b(s)|), s \in \mathbb{R}_{+}$.

Consider firstly the case $l$ is odd. For odd $m$ and even $n$, the parameterization (6.5) has an selfintersection real point: $\psi_{0 l}\left(s_{1}\right)=\psi_{0 l}\left(s_{2}\right)$ for $s_{1} \in\left(-\frac{n-l}{m-l},-\frac{n}{m}\right), s_{2} \in(0, \infty)$. In all other cases the image of $\psi_{0 l}\left(\mathbb{R}_{+}\right)$lies in the quadrant which does not contain any other real branches of $\psi_{0 l}$ restricted on $\mathbb{R}_{-}$. Detailed analysis shows that the real branch of $\psi_{0 l}\left(\mathbb{R}_{+}\right)$lies in

(1) quadrant $\{a<0, b<0\}$ for odd $m$ and $n$; 
(2) quadrant $\{a>0, b>0\}$ for even $m$ and $n$;

(3) quadrant $\{a>0, b<0\}$ for even $m$ and odd $n$.

Therefore, the corresponding reflections are the following:

$$
(1)(a, b) \rightarrow(-|a|,-|b|),(2)(a, b) \rightarrow(|a|,|b|),(3)(a, b) \rightarrow(|a|,-|b|) .
$$

Now consider the case when $l$ is even. In this case $\psi_{0 l}$ does not take real values for $s \in \mathbb{R}_{+}$. Using the transformation $\psi_{0 l}=(a, b) \rightarrow\left(\varepsilon^{m} a, \varepsilon^{n} b\right)$ with $\varepsilon=(-1)^{\frac{1}{l}}$, we get a new parametrization with two real values on $\mathbb{R}_{+}$. Choose the positive one. In this case, we do not have selfintersection points because the values of $\psi_{0 l}$ for $s \in\left(-\frac{n-l}{m-l},-\frac{n}{m}\right)$ reflect to non real part of $\mathbb{C}^{2}$ and thus for arbitrary $m$ and $n$ the image of $\psi_{0 l}\left(\mathbb{R}_{+}\right)$does not intersect any other real branches of $\psi_{0 l}$. Using the property (1.7) for $\lambda_{0}=a_{0}=1$, $\lambda_{1}=\varepsilon=(-1)^{\frac{1}{l}}, a_{l}=-1, a_{m}=|a|, a_{n}=|b|$ we obtain

$$
\Delta_{0 l}\left(\varepsilon^{m}|a|, \varepsilon^{n}|b|\right)=\Delta\left(1, \lambda_{1}^{l} \cdot(-1), \varepsilon^{m}|a|, \varepsilon^{n}|b|\right)=\varepsilon^{m n} \Delta(1,-1,|a|,|b|) .
$$

Summarizing the above, we get

Statement 6.5. If $l$ is odd, then the convergence domain $D_{0 l}$ is of type

$\left\{\Delta_{0 l}(-|a|,-|b|) \lessgtr 0\right\}$ for odd $m$ and $n$;

$\left\{\Delta_{0 l}(|a|,|b|) \lessgtr 0\right\}$ for even $m$ and $n$;

$\left\{\Delta_{0 l}(|a|,-|b|) \lessgtr 0\right\}$ for even $m$ and odd $n$.

If $l$ is even, then the convergence domain $D_{0 l}$ is of type

$\{\Delta(1,-1,|a|,|b|) \lessgtr 0\}$.

Symmetrically, one has the following result for the domain $D_{m n}$.

Statement 6.6. If $n-m$ is odd, then the convergence domain $D_{m n}$ is of type

$\left\{\Delta_{m n}(-|a|,-|b|) \lessgtr 0\right\}$ for even $l$, even $m$ and odd $n$;

$\left\{\Delta_{m n}(|a|,|b|) \lessgtr 0\right\}$ for even $l$, odd $m$ and even $n$;

$\left\{\Delta_{m n}(-|a|,|b|) \lessgtr 0\right\}$ for odd $l$, even $m$ and odd $n$.

If $n-m$ is even, then the convergence domain $D_{m n}$ is of type

$\{\Delta(|a|,|b|,-1,1) \lessgtr 0\}$.

\section{Funding}

The authors were supported by the Russian Science Foundation N. 20-11-20117.

\section{Disclosure statement}

No potential conflict of interest was reported by the author(s). 


\section{References}

[1] Horn J. Über die Konvergenz der hypergeometrischen Reihen zweier und dreier Veränderlichen. Math. Ann., 1889; Vol. 34: P. 544-600.

[2] Birkeland R. Über die Auflösung algebraischer Gleichungen durch hypergeometrische Funktionen. Math. Zeitschrift, 1927; Bd. 26. Tl. 1: S. 565-578.

[3] Mellin Hj. Resolution de l'equation akegbrique generale a l'aide de la function gamma. C.R. Acad. Sci. Paris, Ser. 1 Math, 1921; Vol. 172: P. 658-661.

[4] Sturmfels B. Solving algebraic equations in terms of A-hypergeometric series. Discrete Math., 2000; Vol. 210, iss. 1/3: P. 171-181.

[5] Passare M., Tsikh A.K. Algebraic equations and hypergeometric series. The Legacy of N.H. Abel, Springer-Verlag, 2004; P. 653-672.

[6] Nilsson L., Passare M., Tsikh A.K. Domains of convergence for A-hypergeometric series and integrals. J. of Siberian Federal University, Math. and Phis., 12:4 (2019) P. 509-529.

[7] Antipova I.A., Mikhalkin, E.N. Analytic continuation of a general algebraic function by means of Poiseaux series. Steklov Inst. Math., 279 (2012), 1, P. 3-13.

[8] Dickenstein A., Sadykov T. English translation: Basis in the solution space of the Mellin system, Sbornik: Mathematics (2007), 198(9), 1277-1298.

[9] Zhdanov O.N., Tsikh A.K. Investigation of multiple Mellin-Barnes integrals by means of multidimensional residues. (Russia) Sibersk. Mat. Zh. 39 (1998), 2, 281-298, i: translation in Siberian Math. J. 39 (1998), 2, 245-260.

[10] Antipova I.A. An expression for the superposition of general algebraic finctions in terms of hypergeometric series. (Russia) Sibirsk. Mat. Zh. 44 (2003), 5, 972-980, i: translation in Siberian Math. J. 44 (2003), 5, 757-764.

[11] Nguyen Thanh Hai, Yakubovich S.B. The double Mellin-Barnes type integrals and their applications to convolution theory. Series of Soviet and East Europian Mathematics, 6 . World Scientific Publishing Co., Inc., River Edge, NJ, 1992, X+295 pp.

[12] Antipova I.A., Mikhalkin, E.N., Tsikh A.K. Rational expressions for multiple roots of algebraic equations. Mat. Sb. 209 (2018), no. 10, 3-30; translation in Sb. Math. 209 (2018), no. 10, 1419-1444.

[13] Gelfand I.M., Kapranov M.M., Zelevinsky A.V. Discriminants, resultants and multidimensional determinants. Birkhäuser, 1994.

[14] Passare M., Sadykov T.M., Tsikh A.K. Nonconfluent hypergeometric functions in several variables and their singularities. Compos. Math. 2005. Vol. 141, iss. 3. P. 787-810.

[15] Passare M., Tsikh A.K. Amoebas: Their spines and their contours. Idempotent Mathematics and Mathematical Physis: Intern. Workshop, February 3-10, 2003, Vienna, Austria / Eds. G.L. Litvinov, V.P. Maslov / AMS Contemporary Math., 2005. Vol. 377.

[16] Kapranov M.M., A characterization of A-discriminantal hypersurfaces in terms of the logarithmic Gauss map. Mats. Ann., 1991: Vol.290: P. 277-285. 